\title{
Coal and Gas Outburst Prevention in Longwalls by Means of Powered Support Unit of New Type
}

\author{
Vladimir Tarasov ${ }^{1}$, and Anatoly Fomin ${ }^{2, *}$ \\ ${ }^{1}$ Rivals Modern Innovative Technologies LLC \\ 2 JSC NC VOSTNII, 6500023 Institutskaya st., Kemerovo, Russia
}

\begin{abstract}
The paper considers a new type of powered roof support unit in the framework of interaction with geomechanical processes within the ground rock, particularly, in the thermodynamic balance capsule. Its novelty in comparison with similar Russian and foreign technologies consists is in the fact that geomechanical system "support-ground rock" is brought into the state of balance, which increases the safety of mining works at a coal extraction face.
\end{abstract}

\section{Introduction}

Investigation of the causes of accidents associated with the sudden outburst of coal, dust and gas mixture reveals major problems, identifies gaps in scientific research and practical approaches to ensuring miners' safety in the extraction of coal by deep mining. It is for this reason that Russian and foreign scientists are focused on the research to reduce the level of accidents and injuries at coal enterprises [1-8].

\section{Materials and Methods}

A new type of powered support unit (PSU) is considered in terms of interaction with geomechanical processes in the ground, namely, the thermodynamic balance capsule (TDBC).

The use of movable hydraulic-controlled valve in the wall rock and the lock in TBDC for the extraction of solid minerals (coal) by the deep mining method with PSU of a new type has been unknown to the scientific community and it has never been applied in practice. We will analyze and compare this type of roof support with such types as chockshield support, shield powered support and shield-chock support.

There is an analogue of the PSU of the shield-chock type, which is used to build theoretical conclusions based on the laws of theoretical mechanics, physics and geomechanics.

After mounting and launching the operation of powered roof support in the longwall during the primary collapse of the main roof in a goaf part of the longwall, the face space

\footnotetext{
* Corresponding author: fominai@kuzstu.ru
} 
and the face itself are located in the center of the first zone of the TDBC while mining the extraction pillar (the gas content in the longwall must not exceed the norms).

At the exit from the longwall, the concentration of methane gas must not exceed $1 \%$, while along the longwall local accumulations of methane must not exceed $2 \%$.

The entire volume of methane gas in the longwall is divided into three parts: the first part is the volume contained in the coal broken by a mining machine in one cycle, shift, and day; the second part is the volume that is displaced from the coal seam itself; and the third part is the volume, which is displaced along fractures formed in the interstrata rock mass during the longwall operation coming out of the face space soil from the lower strata. The longwall operates in the center of a "dust bag".

The calculation of the gas content in the longwall is made based on the volume of the first part. Since this volume is controllable, it is possible to increase or decrease the speed of coal cutting by a mining machine [9].

The weakness of the known technical solution is that the technical state of the PSU of chock-shield, shield support and shield-chock types is unable to influence the other two parts of the gas volume (second and third), however, it can be influenced by boundary ventilation, drilling a well from the surface, drilling wells along the longwall column sides, as well as by means of all sorts of expensive degassing measures. These methods are inefficient, since the volume of methane gas for this certain period depends on the geomechanical processes of interaction of the ground with the PSU and the pace of face advancing.

\section{Results and Discussion}

Consider the mutual operation of two structures: the man-made one and the geomechanics of natural processes in the ground in the framework of TDBC [10].

When mounting a powered roof support complex of a shield-chock type in an installation chamber, one can see how the TDBC is formed around the installation chamber of the longwall (Fig. 1). TDBC consists of three zones. The third zone is formed at a distance of two radii, until the radius reaching a length of $29.6 \mathrm{~m}$ - the primary step of the main roof caving, and self-organizes in the installation chamber, circumscribing the face area circle - the shell of the shielding subsystem in the form of a ball, a ring in cross section of rocks with high stress and stiffness, and optimum bearing capacity. All radii are laid off from the center of the installation chamber cross section. Then the third zone acquires an infinite bearing capacity and is located at a distance of $29.6 \mathrm{~m}$ from the nearest edge of even an expanding cavity. This shell isolates the three-zone subsystem from the effects of the stressed ground. This is a zone of high ring stress and high rigidity.

Thus, the process of redistributing stresses around the cavity of the operating longwall creates an elongated TDBC around the longwall, that is, a self-organizing, isolated shielding subsystem to protect the ground rock and the cavity from further destruction. The dome of the natural equilibrium over the cavity can be formed only in the conditions of the first zone of the TDBC - the installation chamber, where there is no ring stress and impact of the stressed ground rock, which is a pattern of ground self-organization around the cavity, the same for all conditions. 


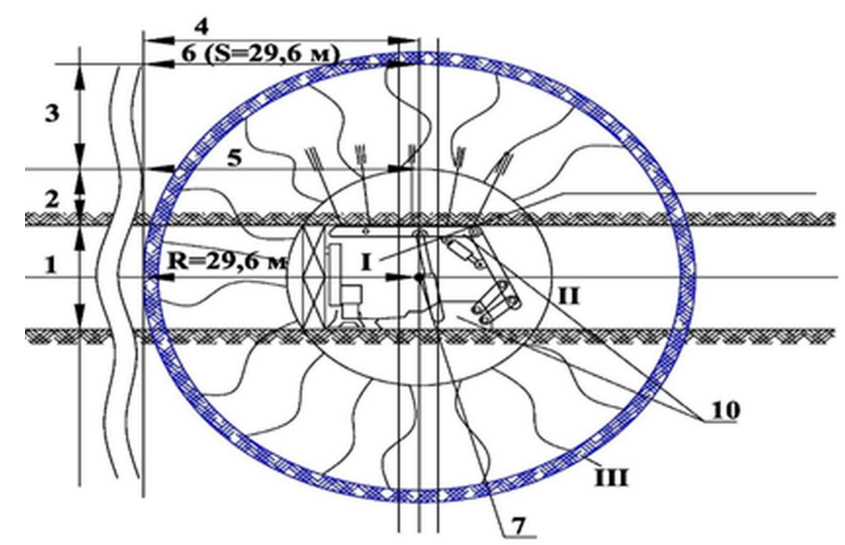

Fig. 1. General view of the PSU in the installation chamber and the formation of TDBC around the installation chamber. I - the first zone of TDBC (the installation chamber and the longwall); II - the second zone of TDBC; III - the third zone of TDBC (the shielding shell of the subsystem, a ring 100$150 \mathrm{~mm}$ wide); 1 - a solid mineral (coal) stratum; 2 - immediate roofing; 3 - the main roof; 4 -zone of the subsequent primary step of roof rock caving; 5 - the step of the immediate roof caving; 6 - the step of the main roof caving; 7 - the center of TDBC, from which the diameters and radii of all zones are laid off, 10 - PSU.

As shown in Figure 2, coal seam 1 transfers all the ring loads to the third zone III and is itself subjected to deformation, which leads to an uncontrolled release of the second part of the methane gas volume $\mathrm{V}_{2}$. The face line is on the diameter of all three zones, the center of the TDBC is shifted from the center of the installation chamber to the vertical face line, the face area is almost in the center of the "gas bag", which leads to tragic outcomes such as those which occurred in "Ulyanovskaya", "Raspadskaya" and "Severnaya" mines.

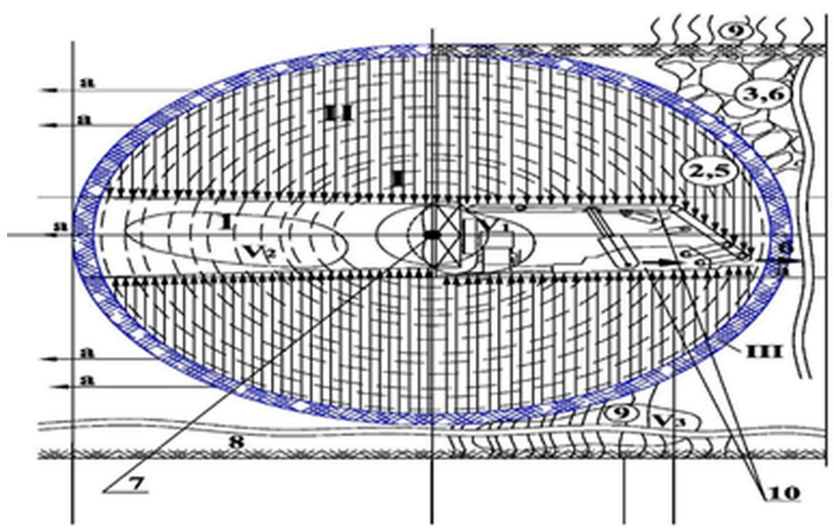

Fig. 2. Operation of the PSU in the TDBC under the current technology. $a$ - the direction of face advancing and the movement of TDBC ahead of the face; $b$ - the direction of the PSU departure to the caving; 8 - wall rocks; 9 - perpendicular parallel lines, cracks; $V_{1}$ - the volume of methane gas from the coal broken by the mining machine and blowing from the fresh face; $V_{2}-$ the volume of methane gas displaced from the seam clamping; $\mathrm{V}_{3}$ - the volume of methane emerging from the underlying strata along parallel vertical cracks. 
Figure 2 shows how PSU 10 cannot cope with its task and cannot resist the pressure of the rock enclosed in the second zone II at the proper level. The PSU is advancing amorphously and fulfills only its shielding capacity, while its chocking ability does not manifest itself in any way, it is virtually clamped and pushed from the face line towards caving, or towards the face.

The weakness in the operation of known types of PSU is that the solid mineral (coal) mass does not transmit the primary stress (energy) of the rock that used to be in place of the face area, and the stress of the second zone rock II is not transmitted to the that of the third zone III.

This process is clearly visible when the longwall begins to operate for the excavation of solid minerals (coal) and moves away from the installation chamber and, in the process of longwall advancing, one of the installation chamber walls becomes the face of the longwall. During the first cycles of coal mining, the face is well-oriented, the solid mineral is hard (hard coal), and the machine cuts the coal seam in the face with effort. Along the cutting line, energy is released ahead of time, there is fracturing, cleavage of the ground, sloughing, slipping until the primary caving of the immediate and main roof occurs. Subsequently, all the energy at the speed of sound and at the speed of the face advancing is transferred to the rock of the third zone III, which is in an uncontrolled state, with advance by a distance of the radius of the second zone ring II throughout the column and the length of the longwall during its operation.

Another disadvantage is that the third part, the volume of methane gas (Fig. 2), is displaced along fractures formed in the interstrata ground rock during the longwall operation and leaves the soil of underlying strata of the face area. The cavity of the mining space is the longwall itself and the face area enclosed within the phase TDBC. The shell of the TDBC concentrates colossal energy. As the mining space increases, the radius of this phase capsule increases, and the capsule shell approaches the surface. There comes a moment when the ground mass to the surface and in deeper areas cannot withstand the colossal energy concentrated in this shell, and instantly released energy (in the form of wave shocks) cuts the rock mass along several parallel lines of cracks (Fig. 3).

The third part of the methane volume is released through the cracks, which confirms the phase concentric structure of this capsule shell (Fig. 2).

Thus, uncontrolled support pressure with a very high potential of stress is the main negative factor of all systems of deposit development by the deep mining method.

The technical result of the operation of a new type PSU is a movable hydraulic valve in the wall rock and the lock in the TDBC, which allow us to leave the first part of the methane gas volume in calculations of the longwall gas content that can be affected, and to exclude the second and third parts, which cannot be affected, and what us more, to remove the first zone in which the longwall operates from under the influence of the TDBC, and to make all the energy concentrated in the TDBC shell work together with the PSU functioning as a movable hydraulic valve in the wall rocks and the lock, i.e. to use the physical law of "the Second Condition of a solid body Equilibrium." 


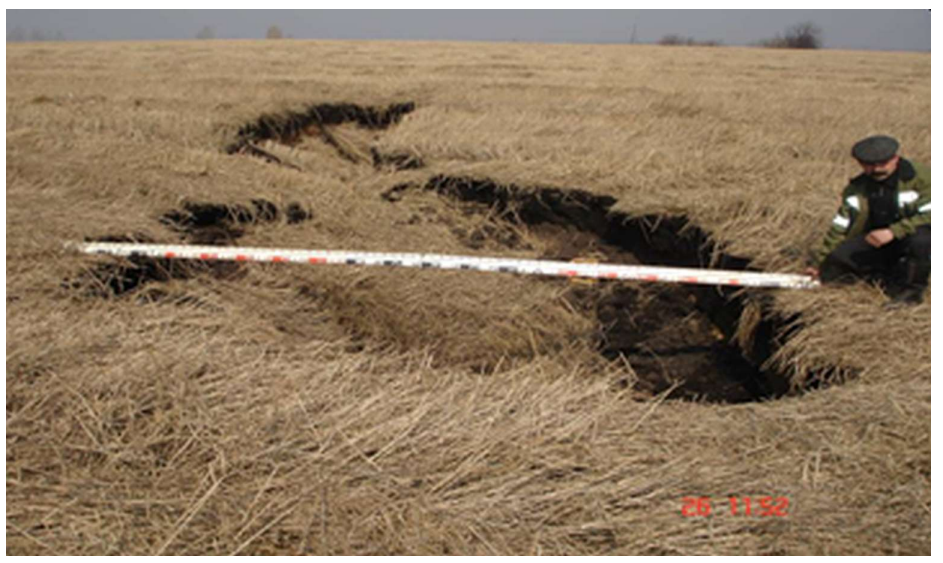

Fig. 3. Rock mass to the surface and in deeper areas cannot withstand the colossal energy concentrated in this shell and instantly released energy in the form of wave shocks cuts the rock mass along several parallel lines of cracks

Figure 4 shows a new type of PSU: a movable hydraulic-controlled valve in the wall rocks and the lock in the TDBC and its operation.

The PSU of the new type - the movable hydraulic valve in the wall rocks and the lock in the TDBC - works as follows. The functions of the valve and the lock in TDBC when unloading the PSU 10 (Fig. 4) of hydraulic leg are reduced. The supporting element performs a non-zero work of the movable hydraulic lock in TDBC (Fig. 4, red colour), the lock opens, the movable hydraulic valve opens slightly (Fig. 5, green colour), and instantly all the colossal energy concentrated in the capsule and the thermodynamic balance shell (Fig. 4, blue colour, zone III and zone II) affects movement of the powered support unit.

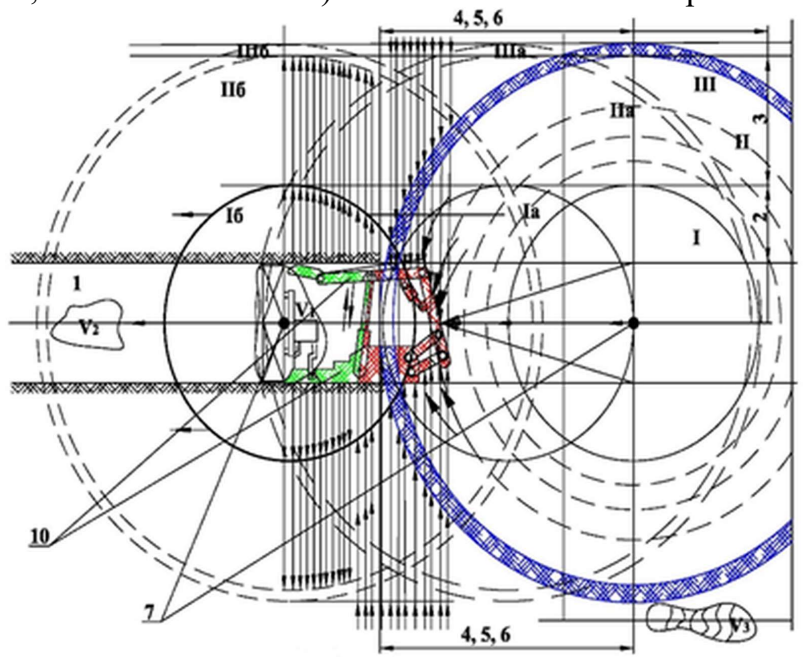

Fig. 4. PSU of a new type: movable hydraulic-controlled valve in wall rocks and the lock in TDBC. Ia - displacement of the TDBC first zone; $\mathrm{Ib}$ - withdrawal of the first zone and its removal from the effect of the TDBC; IIa and IIb - non-displaced zones, the second zone remained in TDBC; IIIa and IIIb - the third zone, non-displaced TDBC zones. 
When a collapse occurs in the goaf, PSU 10 moves back in, a thrust in hydraulic leg occurs, the movable hydraulic valve (green) closes and the movable hydraulic lock (red) in the thermodynamic balance capsule also closes, resulting in the capsule ring (blue) closing through the powered support unit 10 .

The sum of the moments of all external forces acting on it relative to the axis passing through the location of supporting elements and bases and the hydraulic legs themselves is zero: the overpressure in the thrust system of the hydraulic legs of the powered roof support unit is relieved out through the safety valve.

Thus, the second condition for the solid body equilibrium is a condition of zero work, which is performed in the PSU of a new type, a movable hydraulic valve in the wall rocks and the lock in the TDBC.

The power component of the hydraulic leg (lever support reaction) works tangentially to the power component of the third zone III, almost vertically, but they never occupy a vertical position in accordance with Rivals theorem "Motion of a spherical solid relative to a point". Wedges-cutters on the powered roof support unit in the second zone II jointly with the mass of the rock and the forces enclosed in the TDBC itself do work with a positive moment relative to the vertical axis of the lever (Fig. 5), thereby initiating the collapse in the hard-to-die wall rocks. It can be seen how a four-bar linkage with the shield element and the goaf part of the canopy and goaf console arm plays the role of a movable hydraulic lock in TDBC, when unloading PSU 10, the KTDB is torn (Fig. 4). The forces contained in this ring, with the speed of sound, act on the goaf part of the PSU increasing the speed of PSU advancing. When the thrust in PSU occurs, the third zone III ring is closed and the face section of the PSU performs the function of a movable hydraulic valve in the wall rocks, thereby protecting the face space and allowing the first zone I to be withdrawn from the TDBC to the solid all negative effects occurring in the second zone II and outside the TDBC where parallel cracks form in the ground are formed, while the first zone is the face area of the longwall.

\section{Conclusion}

The new type of PSU allows increasing the safety of extraction work, increasing the PSU efficiency, decreasing the gas content and metal consumption of the PSU, rising labour productivity, reducing the cost of coal mining, eliminating emergencies due to a sudden outburst of coal and methane in the longwall.

\section{References}

1. P. A. Ferguson, Min. Cong. J., 12, 211 (1971)

2. German Longwall Mining (Gluckauf GmbH, Essen, 1985)

3. J. Gorrie, M. Scott, Mining and Metallurgical Society of America (West Virginia University, Scottown, 1970)

4. Syd S. Peng, H. S. Chiang, Longwall Mining (Springer, New York,1984)

5. Syd S. Peng, American Coal, 2 (1996)

6. Syd S. Peng, Longwall Mining (West Virginia University, Scottown, 2006)

7. S. Fiscor, Coal Age, 2, 41 (2009)

8. Syd S. Peng. Coal Mine Ground Control (West Virginia University, Scottown, 2008)

9. Guidelines for the design of ventilation of coal mines (MakNII, Moscow, 1989)

10. V. S. Zaburdyaev, Safety in Industry, 12, 70 (2011) 